\title{
Vers de nouvelles recommandations françaises sur la prise en charge des états de mal épileptiques
}

\section{Towards new French Guidelines on the Management of Status Epilepticus}

\author{
V. Navarro $\cdot$ S. Demeret $\cdot$ H. Outin \\ Reçu le 11 avril 2016; accepté le 2 juin 2016 \\ (c) SRLF et Lavoisier SAS 2016
}

Les états de mal épileptiques sont la deuxième urgence neurologique après les accidents vasculaires cérébraux. Leur incidence, bien que difficile à calculer, est estimée à 6-40 nouveaux patients pour 100000 individus par an. Leur mortalité dépend principalement de la cause [1], mais aussi de l'âge du patient et du type d'état de mal : l'état de mal convulsif généralisé étant la forme la plus grave. Les états de mal épileptiques ne doivent pas être considérés comme une pathologie unique, mais plutôt comme le symptôme d'une myriade d'étiologies différentes. Ils ont donc une expression clinique très variée.

Des recommandations détaillées ont été établies en 2008 sous l'égide de la Société de réanimation de langue française [2]. Elles ont porté sur la prise en charge diagnostique et thérapeutique des différents types d'état de mal, par les équipes médicales tant préhospitalières qu'hospitalières. Compte tenu du très faible nombre d'études randomisées et contrôlées, ces recommandations étaient principalement fondées sur des avis formalisés d'experts.

Il est aujourd'hui important de réactualiser de telles recommandations afin d'y inclure : 1) les résultats d'essais thérapeutiques plus récents ; 2) les nouvelles molécules antiépileptiques, disponibles, même si elles n'ont pas encore fait

\footnotetext{
V. Navarro $(\square)$

Unité d'épilepsie et unité d'EEG, Assistance Publique-Hôpitaux de Paris, hôpital Pitié-Salpêtrière et université Pierre et Marie Curie (Paris 6), F-75651 Paris cedex 13 Paris, France

e-mail : vincent.navarro@aphp.fr
}

Institut du cerveau et de la moelle épinière (ICM; INSERM UMRS1127, CNRS UMR7225, UPMC), Paris, France

\section{S. Demeret}

Unité de réanimation neurologique, Assistance Publique-

Hôpitaux de Paris, hôpital Pitié-Salpêtrière et université Pierre et Marie Curie (Paris 6), F-75013 Paris, France

\section{H. Outin}

Service de réanimation médicochirurgicale, CHI Poissy-

St-Germain-en-Laye, F-78303 Poissy, France l'objet d'études prospectives ; 3) les prises de position de sociétés savantes, concernant notamment de nouvelles définitions des états de mal ; 4) l'intérêt de développer des filières de soins spécialisées dans la prise en charge des états de mal ; 5) de nouvelles modalités thérapeutiques : place de l'anesthésie et des traitements immunomodulateurs.

Depuis 2008, un grand nombre d'études a été publié, mais rares sont celles ayant un bon niveau de preuve. L'étude nord-américaine, RAMPART, préhospitalière, a montré que le midazolam, injecté en intramusculaire, était supérieur au lorazépam, injecté en intraveineux [3]. Cette modalité d'injection est probablement plus adaptée au système de santé nord-américain, qui ne bénéficie pas d'équipes médicales préhospitalières. Plus récemment, une étude française multicentrique, SAMUKEPPRA, a montré que le lévétiracétam, injecté en intraveineux, en même temps que le clonazépam, ne permettait pas de contrôler un plus grand nombre de patients [4]. De façon intéressante, les résultats de cette étude randomisée, contrôlée par un placebo, ne vont pas dans le sens des précédentes études, non randomisées ou ouvertes, qui suggéraient l'intérêt du lévétiracétam dans la prise en charge initiale des états de mal. D'autres études, bien que non randomisées, ont fourni des informations intéressantes, qui doivent être validées ; il s'agit principalement d'études testant des traitements antiépileptiques intraveineux en $1^{\text {re }}$ ou en $2^{\mathrm{e}}$ ligne. Les nouvelles recommandations devront donc suivre le format de recommandations d'experts si elles veulent couvrir les principaux champs de prise en charge des états de mal généralisés et focaux.

Un groupe de travail mandaté par la Ligue Internationale contre l'Épilepsie vient récemment de s'intéresser aux définitions de l'état de mal [5]. Les auteurs ont conservé le principe d'une définition « conceptuelle » (ou théorique) et une définition "opérationnelle » adaptée à la présentation clinique (état de mal généralisée tonico-clonique, vs état de mal focal avec troubles de vigilance, vs état d'« absence »). Pour ces différentes formes d'état de mal, un temps tl est défini à partir duquel il est clair qu'il ne s'agit pas d'une crise 
prolongée, et un temps $\mathrm{t} 2$ est défini à partir du moment où le risque de lésion neuronale excitotoxique devient important. Bien que n'étant pas étayée par des arguments cliniques et scientifiques formels, cette proposition de définition a le mérite de souligner la nécessité d'adopter une attitude thérapeutique pondérée au pronostic de chaque type d'état de mal. De plus, une classification des états de mal est proposée suivant quatre axes : la sémiologie, l'étiologie, l'aspect EEG et l'âge. Sur le plan sémiologique, une distinction est faite selon que les états de mal ont ou non une composante motrice. Il est clair que de nouvelles recommandations françaises sont nécessaires, devant à la fois tenir compte de ces propositions, mais aussi en les adaptant pour en simplifier le message et surtout faciliter leur utilisation. Il importe en effet que les réanimateurs, les urgentistes et les neurologues, qui peuvent tous être amenés à prendre en charge des états de mal partagent le même langage et les mêmes attitudes thérapeutiques.

Des recommandations, uniquement fondées sur des niveaux de preuve, viennent tout récemment d'être publiées par la Société américaine d'épilepsie. Elles se sont limitées à la prise en charge des états de mal épileptiques convulsifs chez l'adulte et l'enfant [6]. Ces recommandations n'ont répondu qu'à cinq questions : quels traitements antiépileptiques sont efficaces? Quels sont les effets secondaires en fonction des modalités d'administration? Quelle est la plus efficace des benzodiazépines ? La fosphénytoïne doit-elle être privilégiée à la phénytoïne ? Après combien d'essais de traitement un état de mal devient réfractaire ? De façon intéressante, les auteurs justifient de telles recommandations, sur le principe d'une prise en charge thérapeutique encore trop inégale, avec notamment des patients recevant des traitements inadaptés, ou recevant des traitements adaptés mais à des doses insuffisantes. Une analyse des effets secondaires des traitements a notamment souligné qu'une détresse respiratoire était plus souvent associée à l'administration d'un placebo qu'à l'administration d'une benzodiazépine, montrant que la détresse respiratoire est surtout liée à la durée de l'état de mal. Les recommandations uniquement fondées sur des preuves ont leur limite dans l'état de mal, notamment quand il s'agit de conseiller un traitement de $2^{\mathrm{e}}$ ligne, mais surtout pour ceux de $3^{\mathrm{e}}$ ligne. D'où l'intérêt des recommandations de la Société de neuro-réanimation nord-américaine [7], qui allient des propositions fondées sur les quelques études à haut niveau de preuves, et des avis d'experts quand ces dernières font défaut.

Toutes les recommandations sont également l'occasion d'identifier des attitudes thérapeutiques pour lesquelles des études contrôlées, de haut niveau de preuve, font défaut, une situation très fréquente dans la prise en charge de la diversité des états de mal. C'est le cas de la prise en charge des états de mal partiels et des états de mal réfractaires. Des essais thérapeutiques, en cours ou à venir, devraient répondre à certaines questions. À la phase préhospitalière, une étude nordaméricaine (ESSET), vient de débuter, comparant la fosphénytoïne, le lévétiracétam et le valproate de sodium, chez des adultes ou des enfants, dont l'état de mal convulsif n'a pas cédé après injection de benzodiazépines. En France, une étude préhospitalière (LORACLOFT) est en cours, comparant l'efficacité du lorazépam, du clonazépam seul ou associé à la phosphénytoïne, dans les états de mal convulsifs. Une autre étude française multicentrique (VALSE) porte actuellement sur l'intérêt d'adjoindre du valproate de sodium aux patients admis en réanimation, pour un état de mal convulsif, afin d'en diminuer la mortalité.

Enfin, les recommandations sont aussi une opportunité pour souligner la nécessité du développement de filières de soins spécialisées dans la prise en charge des états de mal, s'appuyant sur un réseau de compétence des différents acteurs, tant préhospitaliers qu'hospitaliers. L'enregistrement continu de l'EEG et de la vidéo, interprété par un neurophysiologiste expérimenté, est, chez un patient présentant un état de mal réfractaire, une des mesures qui devraient clairement améliorer la prise en charge des patients. Or, les réanimations en France ayant accès à un tel environnement médicotechnique sont encore rares. Cet outil est néanmoins à double tranchant : une surveillance continue de l'EEG en réanimation chez un patient cérébrolésé peut mettre en évidence de nombreuses activités pathologiques mais pas nécessairement épileptiques, qu'il faut savoir interpréter avec précaution, pour éviter de traiter par excès ces patients.

La place de l'intubation orotrachéale pour l'administration de molécules anesthésiantes dans la prise en charge des états de mal réfractaires mérite d'être bien discutée. Des études récentes ont montré que la mortalité et la morbidité pouvaient être augmentées par l'anesthésie générale [8]. Les nouvelles recommandations devront rappeler la diversité des formes cliniques d'état de mal, la diversité des pronostics et donc l'attitude graduelle à proposer : par exemple, savoir ne pas intuber et ne pas recourir à une anesthésie chez un patient présentant un état de mal à expression focale, même s'il est réfractaire aux premières lignes de traitement. Dans la prise en charge des états de mal convulsifs généralisés, réfractaires aux premières lignes de traitement, l'attitude thérapeutique n'est pas uniciste : faut-il recourir rapidement à des traitements anesthésiques pour minimiser les risques d'une excito-toxicité neuronale et éviter la pharmacorésistance ? Ou bien faut-il recourir à d'autres traitements antiépileptiques sans réaliser d'intubation?

L'amélioration de la prise en charge des états de mal ne repose pas que sur les traitements antiépileptiques « symptomatiques ». Identifier précocement une cause pour laquelle il existe un traitement " étiologique » demeure une priorité. Dans les états de mal réfractaires de novo (ou « NORSE » en anglais), il convient aujourd'hui d'évoquer systématiquement une encéphalite auto-immune [9], et de proposer 
rapidement un traitement immuno-modulateur, sans attendre la confirmation biologique. Il existe aujourd'hui des faisceaux d'arguments cliniques et paracliniques qui doivent faire rechercher un auto-anticorps connu, mais aussi de suspecter une encéphalite auto-immune alors que les tests actuellement disponibles ne permettent pas d'en définir la cible antigénique.

Liens d'intérêts : Le Pr Navarro déclare avoir fait partie d'un board du laboratoire EISAI, et avoir reçu des rémunérations du laboratoire UCB pour une intervention lors de formations en EEG.

\section{Références}

1. Betjemann JP, Lowenstein DH (2015) Status epilepticus in adults. Lancet Neurol 14:615-24

2. Outin H, Blanc T, Vinatier I (2009) [Emergency and intensive care unit management of status epilepticus in adult patients and children (new-born excluded). Rev Neurol (Paris) 165:297-305
3. Silbergleit R, Durkalski V, Lowenstein D, et al (2012) Intramuscular versus intravenous therapy for prehospital status epilepticus. N Engl J Med 366:591-600

4. Navarro V, Dagron C, Elie C, et al (2016) Prehospital treatment with levetiracetam plus clonazepam or placebo plus clonazepam in status epilepticus (SAMUKeppra): a randomised, double-blind, phase 3 trial. Lancet Neurol 15:47-55

5. Trinka E, Cock H, Hesdorffer D, et al (2015) A definition and classification of status epilepticus--Report of the ILAE Task Force on Classification of Status Epilepticus. Epilepsia 56:151523

6. Glauser T, Shinnar S, Gloss D, et al (2016) Evidence-Based Guideline: Treatment of Convulsive Status Epilepticus in Children and Adults: Report of the Guideline Committee of the American Epilepsy Society. Epilepsy Curr 16:48-61

7. Brophy GM, Bell R, Claassen J, et al (2012) Guidelines for the evaluation and management of status epilepticus. Neurocrit Care 17:3-23

8. Sutter R, Marsch S, Fuhr P, et al (2014) Anesthetic drugs in status epilepticus: risk or rescue? A 6-year cohort study. Neurology 82:656-64

9. Gaspard N, Foreman BP, Alvarez V, et al (2015) New-onset refractory status epilepticus: Etiology, clinical features, and outcome. Neurology 85:1604-13 